\title{
A Simplified Approach for Economic Dispatch with Piecewise Quadratic Cost Functions
}

\author{
S. SUBRAMANIAN, Senior member, IEEE and S. GANESAN
}

\begin{abstract}
This paper presents a simple methodology for solving economic dispatch problems with multiple fuel options. The problem has been solved in two stages. In the first stage, the most economic fuel of each unit is identified and in the second stage Economic Load Dispatch (ELD) for the selected fuels is performed. The Composite Cost Function (CCF) is used to identify the most economical fuel and the feasible operating region of each unit. With these details, Sequential Approach with Matrix Framework (SAMF) is performed to determine the optimal dispatch of each unit. The effectiveness of the proposed methodology is tested with a standard ten unit system. Numerical simulation results indicate that the proposed methodology has close agreement with recent reports.
\end{abstract}

Index Terms-Composite cost function, economic load dispatch, piecewise quadratic cost function, sequential approach.

\section{INTRODUCTION}

The primary objective of the economic load dispatch problem is to schedule the generations of thermal units so as to meet the required load demand at minimum operating cost while satisfying the system constraints. Traditionally, the cost function for generating units has been approximated as a quadratic function. The efforts on solving the economic dispatch problems have employed various mathematical programming methods and optimization techniques. The classical optimization methods are reported that to be highly sensitive to the selection of the starting points and sometimes converge to a local optimum.

The methods based on the operational research and artificial intelligence concepts such as genetic algorithm, evolutionary algorithms, fuzzy and artificial neural networks have been given attention for solving economic dispatch problems because of their ability to find the solution near global optimal. Simulated Annealing (SA) technique is similar to a local search technique had been applied to determine the optimal generation schedule for economic dispatch problem in a power system [1]. Genetic Algorithm (GA), a heuristic search technique has been applied to find the optimal generations of thermal units considering the prohibited operating zones of some generating units [2]. A neural net topology based on multilayer perceptron has been developed to solve economic power dispatch problem [3].

Manuscript received May, 2010.

S. Subramanian is with the Department of Electrical Engineering, Annamalai University, Annamalainagar, Tamilnadu, India. (e-mail: dr_smani@yahoo.co.in).

S. Ganesan is with the Department of Electrical Engineering, Annamalai University, Annamalainagar, Tamilnadu, India. (e-mail: ganeshshriraj@gmail.com).
Two phase neural net model has been developed to determine the optimal schedule of generating units [4].

The heuristic search techniques such as Particle swarm Optimization (PSO), Evolutionary Programming (EP), Differential evolution (DE) and hybrid versions of above mentioned search techniques are also proposed for solving economic dispatch problems. The stochastic search technique, PSO has been developed by the inspiration of bird flicking characteristics and it has been applied to solve the economic dispatch problems considering various generator operational constraints [5]. Three different evolutionary programming techniques such as Classical EP (CEP), Fast EP (FEP) and Improved Fast EP (IFEP) have been developed with the different mutation techniques and the effectiveness are validated for solving different kinds of economic dispatch problems [6]. The viability of PSO has also been tested for solving different types of economic dispatch problems [7].

Differential Evolution (DE) based solutions for economic dispatch problem has also been presented [8]. Variable Scaling Hybrid Differential Evolution (VSHDE) has been developed which overcomes the demerit of the fixed and random scaling factor in differential evolution and the effectiveness has been tested for solving medium and large scale economic dispatch problems [9]. The hybrid search techniques have been applied to obtain the economic schedule of generations [10]-[11]. An approach based on a new coding scheme for solving this type of problems through Simulated Annealing like Particle Swarm Optimization (SA-PSO) has been suggested [10]. Bacterial Foraging algorithm, a new optimization technique has been developed by the inspiration of foraging strategy of E.coli bacteria present in human intestine and it is combined with Nelder-Mead method had also been applied to solve economic dispatch problems [11]. The heuristic search methods provide the optimal solution because the efficiency of their search process which depend the various control parameters. The control parameters such as population size, number of generation, etc are problem independent and the solution quality depends on these control parameters.

In certain fossil fired generating units use different fuels hence the cost function are represented as a segmented piecewise quadratic function. The generating units, particularly those units which are supplied with multiple fuel sources are faced with the problem of determining the most economical fuel to burn. The piecewise quadratic function is approximated as piecewise linear function and solved by the traditional methods. A better approach is needed to retain the assumptions of piecewise quadratic function and to solve this problem. Lin and Viviani [12] have reported a hierarchical method based decentralized computations to solve the 
economic dispatch problem with piecewise quadratic functions. Artificial neural network models such as Hopfield Neural Network (HNN) and Adaptive Hopfield Neural Network (AHNN) models have been presented for the solution of economic load dispatch problems with multiple fuel options [13]-[14]. Baskar et.al, reported Real Coded GA (RCGA) to solve economic dispatch problems with piecewise quadratic cost functions [15]. Recently, Artificial Immune System (AIS) has been applied to solve economic dispatch problems [16]. AIS imitates the immunological ideas and utilizes the clonal selection principle and evolutionary approach wherein cloning of antibodies is performed followed by hypermutation. AIS algorithm has also been applied to solve the economic dispatch problem with piecewise quadratic equations.

In this article, a new methodology based on composite cost function and sequential approach with matrix framework has been presented for solving economic dispatch problems with piecewise quadratic cost functions. The most economic fuel of each generating unit is identified by evaluating the composite cost function of each unit and then by performing sequential approach with a matrix framework. After narrowing the search space, the economic dispatch for the selected fuel is obtained by sequential approach with matrix framework.

\section{PROBLEM FORMULATION}

In the economic dispatch problem, the fuel cost of each generator is represented by a single quadratic cost function. Owing to multiple fuel options, the cost function may become piecewise quadratic. Hence, the economic dispatch problem with piecewise quadratic function is defined as,

$$
\min \sum_{i=1}^{N} F_{i}\left(P_{i}\right)
$$

Where

$$
F_{i}\left(P_{i}\right)=\left\{\begin{array}{l}
a_{i 1} P_{i}^{2}+b_{i 1} P_{i}+c_{i 1}, \text { fuel } 1, P_{i, m i n} \leq P_{i} \leq P_{i 1} \\
a_{i 2} P_{i}^{2}+b_{i 2} P_{i}+c_{i 2}, \text { fuel } 2, P_{i 1} \leq P_{i} \leq P_{i 2} \\
\cdot \\
a_{i m} P_{i}^{2}+b_{i m} P_{i}+c_{i m}, \text { fuel } m, P_{i, m-1} \leq P_{i} \leq P_{i, \max }
\end{array}\right.
$$

Where, $a_{i m}, b_{i m}$ and $c_{i m}$ are the cost coefficients of generating unit $\mathrm{i}$ using the fuel type $\mathrm{m}, \mathrm{N}$ is the number generating units and $\mathrm{P}_{i}$ is the active power generation of generating unit $\mathrm{i}$ in $\mathrm{MW}$.

This constrained optimization problem is subjected to variety of constraints. These include power balance constraints and maximum and minimum power generation limits of generating units. These constraints are discussed as follows.

\section{A. Power balance constraint}

This constraint is based on the principle of equilibrium that the total generation should satisfy the total system demand.

$$
\sum_{i=1}^{n} P_{i}=P_{G}=P_{D}
$$

Where, $\mathrm{P}_{\mathrm{D}}$ is the total load demand of the system in MW.

\section{B. Generating capacity constraints}

The active power output of each generator has a lower and upper bound so that the generations of generating unit lies within this limit. This inequality can be given as follows.

$$
P_{i, \min } \leq P_{i} \leq P_{i, \max }
$$

Where, $\mathrm{P}_{\mathrm{i}, \mathrm{min}}$ and $\mathrm{P}_{\mathrm{i}, \max }$ are the minimum and maximum value of active power allowed at generator $\mathrm{i}$ in $\mathrm{MW}$.

\section{Solution Methodology}

A simplified solution methodology for solving economic dispatch problem with piecewise quadratic cost function has been detailed in this section. This methodology comprises two stages. In the first stage, the most economic fuel of each unit is identified using composite cost function. In second stage, the optimal dispatches of generating units are obtained by sequential approach with matrix framework. The derivation of composite cost coefficients and the methodology of sequential approach with matrix framework are presented in this section. The flow of two stage methodology is shown in Fig. 1.

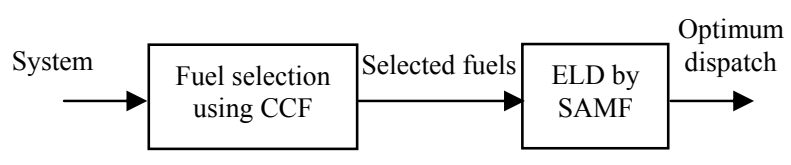

Fig. 1. Two stage methodology

\section{A. Evaluation of composite cost function}

The incremental production cost of a plant is a prior requirement for coordination among plants. The incremental production cost of the plant can be derived by a simple realignment of the fuel cost coefficients of the units.

Consider an ' $n$ ' unit system and the cost equation of $i$ th unit is,

$$
F_{i}=a_{i} P_{i}^{2}+b_{i} P_{i}+c_{i} \quad ; i \in n
$$

and the composite cost function of the plant can be written as,

$$
F_{T}=A P_{G}^{2}+B P_{G}+C
$$

Where, $\mathrm{P}_{\mathrm{G}}$ is the total generation in MW.

The composite cost coefficients are derived as follows.

The total fuel cost $(\$ / \mathrm{h})$ of the plant can be written as,

$$
F_{T}=F_{1}+F_{2}+\ldots . .+F_{n}
$$

For most economical generation,

$$
\begin{aligned}
& P_{i}=\left(\lambda-b_{i}\right) / 2 a_{i} \\
& P_{G}=(\lambda-B) / 2 A_{i}
\end{aligned}
$$

Where, $\lambda$ is the incremental production cost of the plant in MW.

The total generation of the plant can be written as,

$$
\begin{aligned}
P_{G}= & P_{1}+P_{2}+\ldots . .+P_{n} \\
P_{G}= & {\left[(\lambda / 2)\left(1 / a_{1}+1 / a_{2}+\ldots .+1 / a_{n}\right)\right] } \\
& -\left[(1 / 2)\left(b_{1} / a_{1}+b_{2} / a_{2}+\ldots .+b_{n} / a_{n}\right)\right] \\
\lambda= & 2\left[1 /\left(1 / a_{1}+1 / a_{2}+\ldots .+1 / a_{n}\right)\right] P_{G} \\
& +\left(b_{1} / a_{1}+b_{2} / a_{2}+\ldots .+b_{n} / a_{n}\right) \\
& {\left[1 /\left(1 / a_{1}+1 / a_{2}+\ldots .+1 / a_{n}\right)\right] }
\end{aligned}
$$


By comparing Eq. (8) and Eq. (9),

$$
\begin{aligned}
& A=1 /\left(1 / a_{1}+1 / a_{2}+\ldots .+1 / a_{n}\right) \\
& B=\left(b_{1} / a_{1}+b_{2} / a_{2}+\ldots .+b_{n} / a_{n}\right) A
\end{aligned}
$$

The fuel cost can be rewritten as,

$$
\begin{aligned}
& F_{i}=\lambda^{2} / 4 a_{i}-b_{i}^{2} / 4 a_{i}+c_{i} ; \\
& F_{T}=\lambda^{2} / 4 A-B^{2} / 4 A+C ;
\end{aligned}
$$

From Eq. (12),

$$
\begin{aligned}
C= & \left(c_{1}+c_{2}+\ldots .+c_{n}\right)- \\
& \left(b_{1}^{2} / a_{1}^{2}+b_{2}^{2} / a_{2}^{2}+\ldots .+b_{n}^{2} / a_{n}^{2}\right)+B^{2} / 4 A
\end{aligned}
$$

\section{B. Economic dispatch solution using SAMF}

Sequential approach with matrix framework has been developed for solving economic dispatch problems. A maiden attempt has been made to determine the optimal generation dispatches of a system for all possible load demands in single execution. The demonstration of the solution methodology is presented in this section.

The electric power production in a power plant is allowed to vary from minimum technical limit $\left(\mathrm{P}_{\min }\right)$ to maximum technical limit $\left(\mathrm{P}_{\max }\right)$. Initially the $\mathrm{P}_{\mathrm{i} \text {,min }}$ of all generating units in a power plant are considered as initial state input values and is represented by a single dimensional matrix as,

$$
s=\left[P_{1, \min } P_{2, \min } P_{3, \min }, \ldots \ldots, P_{n, \min }\right]
$$

Based on the above single dimensional matrix, a square matrix (I) is developed to identify the economic schedule of generation. The formation of the square matrix is as follows. The process starts with a step increment in generation by $\Delta$ MW in $\mathrm{P}_{1, \min }$ by keeping the remaining units at its input value. This will form first row of the square matrix.

$$
I_{1}=\left[P_{1, \text { min }}+\Delta P_{2, \text { min }} P_{3, \text { min }}, \ldots \ldots, P_{n, \min }\right]
$$

The increment in generation is made in the second element by keeping the other elements at its input value that leads to the development of second row of the square matrix.

$$
I_{2}=\left[P_{1, \text { min }} P_{2, \text { min }}+\Delta P_{3, \text { min }}, \ldots \ldots, P_{n, \text { min }}\right]
$$

In the same logic, an increment is made for remaining units one at a time and a square matrix with a dimension equal to the number of units has been developed. For every step increment in the operating range of the plant the unit one at a time is allowed to experience the change in generation thus leads to the formation of the square matrix.

$$
I=\left[\begin{array}{l}
I_{1} \\
I_{2} \\
\cdot \\
I_{n}
\end{array}\right]=\left[\begin{array}{cccc}
P_{1, \text { min }}+\Delta & P_{2, \text { min }} & \ldots & P_{n, \text { min }} \\
P_{1, \text { min }} & P_{2, \text { min }}+\Delta & \ldots & P_{n, \text { min }} \\
\cdot & \cdot & \cdot & \cdot \\
P_{l, \text { min }} & P_{2, \text { min }} & \ldots & P_{n, \text { min }}+\Delta
\end{array}\right]
$$

Each element in the square matrix represents the generation of a unit corresponding to the column that should satisfy the unit capacity constraints.

In the square matrix the unit generations of each row that satisfy the constraints are identified and total fuel cost of generation is evaluated. The desired economic schedule of generation is identified by analyzing fitness of each row. The fitness function of each row is calculated as,

$$
f i t(j)=\frac{F_{T}(j)}{(p d+\Delta)} \quad j=1,2, \ldots n
$$

Where, pd is the total of input values.

The schedule with the minimum fitness is chosen as the successive state input values. This process is repeated till all the generating units reach their maximum generation capacity. The feasible solutions for every increment from $\mathrm{P}_{\min }$ to $\mathrm{P}_{\max }$ are obtained and hence the best solution for any load demand falls in the operating boundary can be easily sited.

The detailed computational flow of the proposed method is presented in Fig. 2. The proposed methodology in the form of matrix framework to support the demonstration is as follows.

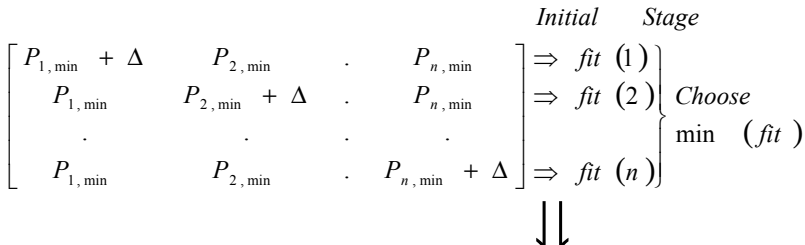

$$
\begin{aligned}
& \text { Final Stage }
\end{aligned}
$$

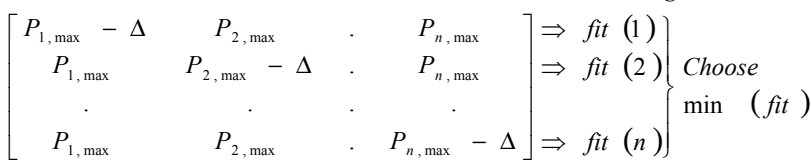

\section{IMPLEMENTATION OF THE PROPOSED METHOD}

The economic dispatch problem with multiple fuel options has been solved in two stages. The implementation procedure of the proposed methodology has been detailed in this section.

\section{A. Stage I: The most economical fuels identification}

The generating units are consists of multiple fuels, each fuel comprises with different cost functions and it is important to identify the most economic fuel of each generating unit for the economic operation of the system. In this section, a new approach has been developed to realize the most economic fuel of generating unit.

The primary search process calculates the composite cost function of each generating unit as detailed earlier section and then SAMF has been performed to identify the most economic fuel of each unit. The composite cost function and unit active power generation limits are considered and sequential approach with matrix framework is performed. The unit generations are the output which indicates the most economic fuel of each unit. The fuel corresponding to the unit generation is the most economical fuel. The generation limit corresponding to the most economic fuel is the feasible operating region of the unit. This search process provides the most economic fuel and the feasible operating region of each unit for all possible load demands.

\section{B. Stage II: Evaluation of optimal dispatch}

In this stage, a secondary search process has been implemented to refine the dispatches of generating units within the feasible operating region. The cost function of the selected fuel of each unit and the corresponding generation 
limits are considered. Based on these details, SAMF has been performed once again to determine the optimum generation dispatch of each generating unit for a particular load demand.

The implementation procedure for solving economic dispatch problem with multiple fuel options is presented in Fig. 3.

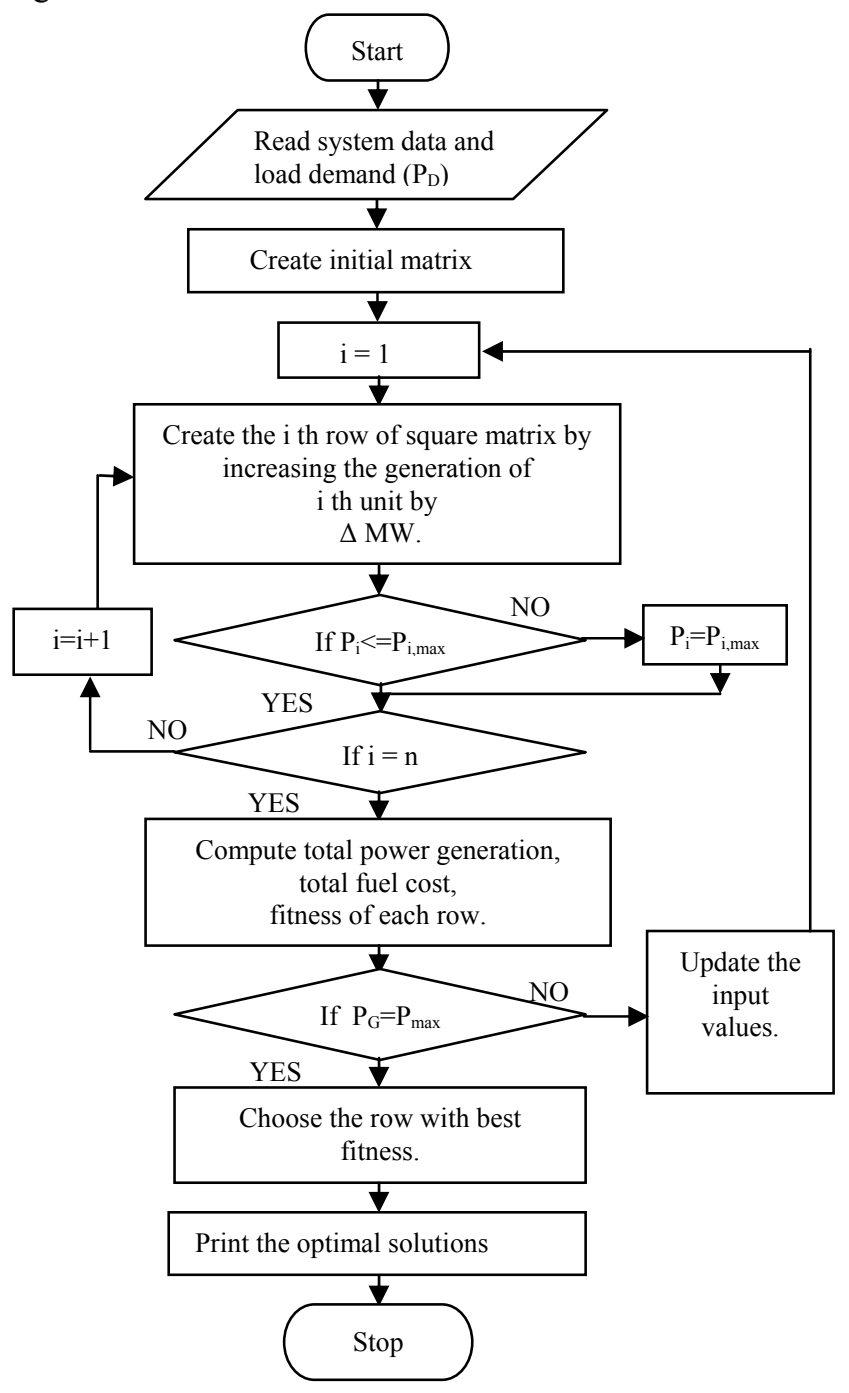

Fig. 2. Computational flow of SAMF

\section{Simulation RESUlts AND Discussions}

The applicability and viability of the proposed approach for practical applications have been tested with a system consists of ten generating units, each generating unit with two or three fuel options [12]. The prime search process has been made to identify the most economic fuel of generating units by the composite cost function and SAMF. After identification of the most economic fuel and the feasible operating regions of each unit, the secondary search process has been achieved to determine the optimal dispatches of generating units. Sequential approach provides the optimal schedule of generations for all possible load demands which is varied from minimum technical limit by a small increment to maximum technical limit of the system. The selection of increment is also an important factor. Too large increment may end up with unfeasible solution and too small increment may take long execution time. Based on experience, the desired increment is chosen as $1 \mathrm{MW}$. The proposed methodology for solving this problem were implemented in Matlab 7.0 platform and executed with Pentium IV, $2.8 \mathrm{GHz}$ personal computer.

This economic load dispatch problem includes the total fuel cost function as an objective with generation of individual units as variables subject to power balance constraint and maximum and minimum generation limits of generating units constraints (i.e. 1 equality and 20 inequality constraints).

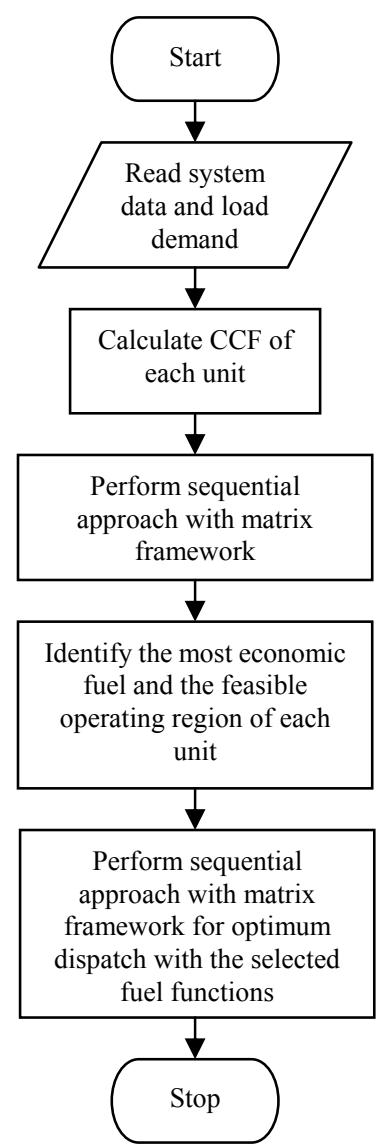

Fig. 3. Computational flow of the proposed methodology

In the first stage, the fuel options of ten individual units are considered as the variables to be optimized. The composite cost function of each generating unit is calculated by combining the cost coefficients of the fuels used in the generating unit. The generation limits and composite cost function of each generating unit are used and the sequential approach is performed. The various combinations of generation dispatches are obtained and the solution quality is analyzed by fitness function. The fitness function is defined as a ratio of total composite cost to total generation. The minimum fitness is considered as optimal. The active power generation of each unit is the output and the fuel corresponding to the generation is the most economical fuel of the unit. The generation limit corresponding to the most economic fuel is the feasible operating region of the unit. This search process provides the most economic fuel of generating units and feasible operating limits for all possible loads.

In second stage, the optimum generations of individual units are obtained within the feasible generation limits through sequential approach. The economical fuel and the 
feasible operating region of each generating unit for a particular demand are considered and sequential approach is performed once again to obtain the optimal dispatches of generating units.

The simulation results for load demands of $2500 \mathrm{MW}$, $2600 \mathrm{MW}$ and $2700 \mathrm{MW}$ are detailed in Table 1. The total fuel cost for above mentioned load demands obtained by this methodology, Hierarchical Method (HM) [12], Hopfield Neural Network (HNN) [13], Adaptive Hopfield Neural Network (AHNN) [14], Hybrid Genetic Algorithm (HGA) [15], Classical Evolutionary Programming (CEP) [6], Fast Evolutionary Programming (FEP) [6], Improved Fast Evolutionary Programming (IFEP) [6], Particle Swarm Optimization (PSO) [7] and Artificial Immune System (AIS) [16] are compared and the comparison of results are detailed in Table 2. The execution time of the proposed methodology for above mentioned load demands is $0.255 \mathrm{~s}$.

TABLE 1ECONOMIC DISPATCH RESULTS

\begin{tabular}{|c|c|c|c|c|c|c|}
\hline \multirow{3}{*}{ Unit } & \multicolumn{6}{|c|}{ Load demand (MW) } \\
\hline & \multicolumn{2}{|c|}{$2500 \mathrm{MW}$} & \multicolumn{2}{|c|}{$2600 \mathrm{MW}$} & \multicolumn{2}{|c|}{$2700 \mathrm{MW}$} \\
\hline & $\begin{array}{l}\text { Fuel } \\
\text { type }\end{array}$ & $\begin{array}{l}\text { Gen. } \\
\text { (MW) }\end{array}$ & $\begin{array}{l}\text { Fuel } \\
\text { type }\end{array}$ & $\begin{array}{l}\text { Gen. } \\
\text { (MW) }\end{array}$ & $\begin{array}{l}\text { Fuel } \\
\text { type }\end{array}$ & $\begin{array}{l}\text { Gen. } \\
\text { (MW) }\end{array}$ \\
\hline 1 & 2 & 207 & 2 & 217 & 2 & 218 \\
\hline 2 & 1 & 206 & 1 & 211 & 1 & 212 \\
\hline 3 & 1 & 266 & 1 & 279 & 1 & 281 \\
\hline 4 & 3 & 236 & 3 & 239 & 3 & 240 \\
\hline 5 & 1 & 258 & 1 & 275 & 1 & 278 \\
\hline 6 & 3 & 236 & 3 & 239 & 3 & 240 \\
\hline 7 & 1 & 269 & 1 & 286 & 1 & 288 \\
\hline 8 & 3 & 236 & 3 & 239 & 3 & 240 \\
\hline 9 & 1 & 331 & 3 & 343 & 3 & 428 \\
\hline 10 & 1 & 255 & 1 & 272 & 1 & 275 \\
\hline $\begin{array}{c}\text { Total } \\
\text { cost } \\
(\$ / h)\end{array}$ & \multicolumn{2}{|c|}{526.24} & \multicolumn{2}{|c|}{574.38} & \multicolumn{2}{|c|}{623.81} \\
\hline
\end{tabular}

TABLE 2 COMPARISON OF TOTAL FUEL COST

\begin{tabular}{|c|c|c|c|}
\hline \multirow{2}{*}{ Method } & \multicolumn{3}{|c|}{ Total fuel cost (\$/h) } \\
\cline { 2 - 4 } & $2500 \mathrm{MW}$ & $2600 \mathrm{MW}$ & $2700 \mathrm{MW}$ \\
\hline HM [12] & 526.70 & 574.03 & 625.18 \\
\hline HNN [13] & 526.13 & 574.26 & 626.12 \\
\hline AHNN [14] & 526.23 & 574.37 & 626.24 \\
\hline HGA [15] & 526.24 & 574.38 & 623.81 \\
\hline IFEP [6] & 526.25 & --- & --- \\
\hline FEP [6] & 526.26 & --- & --- \\
\hline CEP [6] & 526.25 & --- & --- \\
\hline PSO [7] & --- & --- & 623.88 \\
\hline AIS [16] & 526.24 & 574.38 & 623.81 \\
\hline $\begin{array}{c}\text { Proposed } \\
\text { method }\end{array}$ & $\mathbf{5 2 6 . 2 4}$ & $\mathbf{5 7 4 . 3 8}$ & $\mathbf{6 2 3 . 8 1}$ \\
\hline
\end{tabular}

From the comparison of results, it is clear that the proposed approach provides comparable result for economic dispatch problem with piecewise quadratic function. The proposed method has always provided better results than all the other methods except HGA and AIS. The optimal fuel cost obtained through the proposed method is exactly same as HGA and AIS. However, when compared with the earlier reported techniques, it is worth pointing out that the computational procedure of the proposed approach for economic dispatch of units with multiple fuel options is minimal.

\section{CONCLUSION}

This paper presents a simplified methodology, combining the subunits to a composite function followed by sequential approach for solving economic dispatch of generating units with piecewise quadratic cost functions. The problem has been solved in two stages. In the first stage the decentralized configuration of generations are considered. The composite cost function of each generating unit is calculated then prime search process is performed to obtain the most economical fuel and the feasible operating region of each unit. In second stage, based on the details of first stage, the optimum generations of individual units are determined by sequential approach. The developed algorithm has been validated with ten unit sample test system, each unit with two or three fuel options. The proposed approach yields the optimal generation dispatch with less computational effort. The comparison of results depicts that the proposed method is efficient to provide the global optimal or high quality solution for economic dispatch problem with piecewise quadratic cost functions.

\section{ACKNOWLEDGMENT}

The authors gratefully acknowledge the management the support and facilities provided by the authorities of Annamalai University, Annamalainagar, India to carry out this research work.

\section{REFERENCES}

[1] K.P. Wong, and C.C. Wang, "Simulated annealing based economic dispatch algorithm", IEE Proc. Gen. Transm. Distrib., vol. 140, pp. 509-515, Nov 1993.

[2] S.O. Orero, and M.R. Erving, "Economic dispatch of generators with prohibited operating zones: a genetic algorithm approach", IEE Proc. Gen. Transm. Distrib., vol.143, pp.529-534, Nov 1996.

[3] M. Djukanovic, M. Calovic, B. Milosevic, and D.J. Sobejic, "Neural-Net based real time economic dispatch for thermal power plants", IEEE Trans. Energy Convers., vol.11, pp.755-761, Dec.1996.

[4] R. Naresh, J. Dubey, and J. Sharma, "Two-phase neural network based modelling framework of constrained economic load dispatch", IEE Proc. Gen. Transm. Distrib., vol.151, No.3, pp. 373-378, May 2004.

[5] Z.L. Gaing, "Particle swarm optimization to solving the economic dispatch considering the generator constraints", IEEE Trans. Power Syst., vol.18, no.3, pp. 1187-1195, Aug. 2003.

[6] T. Jayabarathi, K. Jayaprakash, D. N. Jayakumar, and T. Raghunathan, "Evolutionary programming techniques for different kinds of economic dispatch problems", Elect. Power Syst. Res., vol. 73, pp. 169-176, 2005.

[7] D.N. Jeyakumar, T. Jayabharathi, and T. Raghunathan, "Particle swarm optimization for various types of economic dispatch problems", Elect. Power Energy Syst., vol. 28, pp. 36-42, 2006.

[8] Nasimul Noman, and Hitoshi Iba, "Differential evolution for economic dispatch problems", Elect. Power Syst. Res., vol. 78, pp. 1322-1331, 2008.

[9] Ji-Pyng Chiou, "Variable scaling hybrid differential evolution for large scale economic dispatch problems", Elect. Power Syst. Res., vol. 77, pp. 212-218, 2007.

[10] Cheng -Chien Kuo, "A novel coding scheme for practical economic dispatch by modified particle swarm approach", IEEE Trans. Power Syst., vol.23, no.4, pp. 1825-1835, Nov 2008.

[11] B.K. Panigrahi, and V. Ravikumar Pandi, "Bacterial foraging optimization: Nelder -Mead hybrid algorithm for economic load dispatch", IET Gen. Transm. Distrib., vol. 2, no. 4, pp. 556-565, 2008.

[12] C.E. Lin, and G.L. Viviani, "Hierarchical economic dispatch for piecewise quadratic cost functions", IEEE Trans. Power App. Syst., PAS -103(6), pp. 1170-1175, 1984

[13] J.H. Park, Y.S. Kim, I.K. Eom, and K.Y. Lee, "Economic load dispatch for piecewise quadratic cost function using Hopfield Neural Network", IEEE Trans. Power Syst., vol. 8, no. 3, pp. 1030-1038, 1993. 
[14] K.Y. Lee, A.S. Yome, and J.H. Park, "Adaptive Hopfield Neural Networks for economic load dispatch", IEEE Trans. Power Syst., vol. 13, no. 2, pp. 519-526, 1998.

[15] S. Baskar, P. Subbaraj, and M.V.C. Rao, "Hybrid genetic algorithm solution to economic dispatch problem", Int. J. Computers. Elect. Engg, vol. 39, no. 3, pp. 407-419, May 2003.

[16] B.K. Panigrahi, Salik R. Yadav, Shubham Agarwal and M.K. Tiwari, "A clonal algorithm to solve economic load dispatch", Elect. Power Syst. Res., vol. 77, pp. 1381-1389, 2007.

S.Subramanian is born in Maduari, Tamilnadu, India. He received the B.E degree in Electrical and Electronics Engineering and the M.E degree in Power Systems with distinction from Madurai Kamaraj University, Madurai, India in the year 1989 and 1990 respectively. He received the Ph.D degree in Electrical Engineering from Annamalai University, Annamalainagar, India in the year 2001. He is currently with the Department of Electrical Engineering as a Professor. He has guided $5 \mathrm{PhD}$ projects, 30 M.E projects and 20 B.E projects. He has been pursuing research with several faculty members. He has published a book chapter in Advances in Differential Evolution (Springer book series: Studies in computational intelligence). He published 110 research articles in various referred international journals, national journals, international conferences and national conferences. His area of interest includes power system operation and control, design analysis of electrical machines, power system state estimation and power system voltage stability studies. He is a Senior member in IEEE, Fellow of Institution of Engineers and a member of various professional bodies such as System Society of India, India Society for Technical Education and Indian Science Congress Association. His biography has been included in MARQUIS who is who in the world, MARQUIS who is who in Engineering and International Biographical Centre (IBC), UK. He received the Best Teacher Award in recognition of his research contributions in Annamalai University.

S.Ganesan is born in Pondicherry, India. He received the B.E degree in Electrical and Electronics Engineering from Government College of Engineering, Salem, India in 2002 and M.E degree in Power Systems with distinction in the year 2008 from Annamalai University, Annamalainagar, India. He is currently with the Department of Electrical Engineering as a Lecturer (Senior Scale). He is currently pursuing Ph.D degree (Part-time) in the Department of Electrical Engineering, Annamalai University, Annamalainagar, India. His research topics include power systems operation and control. He is a Life member of India Society for Technical Education. 